\section{SIGNIFICADOS DEL ESPACIO PÚBLICO EN CONTEXTOS DE TRANSFORMACIÓN POR DESASTRES SOCIONATURALES ${ }^{1}$}

Héctor Berroeta ${ }^{2}$, Laís Carvalho ${ }^{3}$ y Andrés Di Masso $^{4}$

\section{Resumen}

Los desastres socionaturales y las acciones de reconstrucción activan la conciencia del entorno construido y la circulación de narrativas en los habitantes de las comunidades afectadas. En este proceso se articulan distintos significados espaciales, que al igual que los grandes discursos del espacio público, operan significando el espacio urbano y condicionando las prácticas de apropiación. Este estudio analiza los relatos de personas afectadas por tres de los desastres socionaturales que ocurrieron en los últimos años en Chile: el terremoto del año 2007 en Tocopilla, la erupción del volcán Chaitén en 2008 y el terremoto y tsunami de 2010

\section{MEANINGS OF PUBLIC SPACE WITHIN THE CONTEXT OF TRANSFORMATIONS TRIGGERED BY SOCIO- NATURAL DISASTERS ${ }^{1}$}

Héctor Berroeta², Laís Carvalho ${ }^{3}$, Andrés Di $\mathrm{Masso}^{4}$

\section{Abstract}

Socio-natural disasters and reconstruction raise awareness of the built environment and generate narratives among the dwellers of affected communities. This is a process that combines different spatial meanings which, as in the case of major discourses on public space, is based on the signification given to the urban space and the conditioning of appropriation practices. This research analyzes the narrations provided by the victims of three socio-natural disasters that have affected Chile in recent years: the 2007 Tocopilla earthquake, the eruption of Chaitén in 2008 and the 2010 Maule earthquake and tsunami. In 
en la región centro sur. Con el objetivo de explorar la configuración de los significados en torno al espacio público en personas que han estado expuestas a los procesos de transformación urbana producidos por el desplazamiento o reconstrucción de barrios tras estos desastres, se realizaron 17 grupos focales con la participación de 117 habitantes de las localidades de Chaitén, Constitución, Dichato y Tocopilla. Desde una perspectiva cualitativa, se analizaron los relatos que configuran las distintas perspectivas discursivas del espacio público: el espacio público como perdido, como agente de la civilidad y como contestación para la consecución de justicia social.

\section{PALABRAS-CLAVE: ESPACIO PÚBLICO; DESASTRES SOCIONATURALES; SIGNIFICADOS ESPACIALES; DISCURSO; CIUDADES CONTESTADAS.}

Recibido: 29-09-2015.

Aceptado: 16-05-2016.

1 Investigación FONDECYT nro. 11121596: “Vínculos socioespaciales en contextos de transformación urbana producida por catástrofes naturales".

2 Chile. Psicólogo, Doctor en Espacio Público y Regeneración Urbana por la Universidad de Barcelona. Profesor titular del Departamento de Psicología Social, Escuela de Psicología y Director del Magister en Psicología Social de la Universidad de Valparaíso. Correo electrónico: hector. berroeta@uv.cl.

3 Chile. Psicóloga por la Pontificia Universidade Católica de São Paulo, Brasil. Candidata a Doctora en Psicología por la Pontificia Universidad Católica de Chile, Escuela de Psicología. Correo electrónico: Icarvalho@uc.cl.

4 España. Doctor en Psicología por la Universidad de Barcelona. Profesor lector en la Universidad de Barcelona, Departamento de Psicología Social, Facultad de Psicología. Correo electrónico: adimasso@ub.edu. order to compile the meanings given to the public space by those who have experienced urban transformation processes as the consequence of displacement or post-disaster reconstruction, this research conducted 17 focus groups with 117 inhabitants from Chaitén, Constitución, Dichato and Tocopilla. From a qualitative perspective, this contribution analyzes the narrations that establish a relationship among the different approaches to the public space: the public space as a lost place, the public space as an agent of civility and as the place to attain social justice.

\section{KEYWORDS: PUBLIC SPACE; SOCIO-NATURAL DISASTERS; SPATIAL MEANINGS; DISCOURSE; CONTESTED CITIES.}

Received: 29-09-2015.

Accepted: 16-05-2016.

1 FONDECYT research No 11121596: “Socio-Spatial Relationships within the Context of Urban Transformations Triggered by Natural Disasters".

2 Chile. Psychologist, PhD in Public Spaces and Urban Regeneration, University of Barcelona. Professor, Department of Social Psychology at the School of Psychology, University of Valparaíso. Director, Master's Program in Social Psychology at the University of Valparaíso. Email: hector. berroeta@uv.cl.

3 Chile. Psychologist, Pontifical Catholic University of São Paulo, Brazil. PhD candidate in Psychology, Pontifical Catholic University, Chile. School of Psychology. Email: Icarvalho@uc.cl.

4 Spain. PhD in Psychology, University of Barcelona. Professor at the Department of Social Psychology, Faculty of Psychology, University of Barcelona.Email: adimasso@ub.edu. 


\section{Introducción}

Los cambios residenciales y las transformaciones urbanas que se generan a consecuencia de desastres socionaturales ${ }^{5}$, son modificaciones de los hábitat que impactan los vínculos sociales y espaciales de las personas ${ }^{6}$. El proceso de afectación de estos vínculos se configura a partir de la conformación de repertorios de significación y acción sobre el espacio público, mediante los cuales los espacios se conforman en lugar ${ }^{7}$. De acuerdo con Stedman ${ }^{8}$, los significados del lugar son un vínculo intermedio entre las propiedades físicas del lugar y la fuerza del vínculo emocional con él, por lo que para entender los cambios en los vínculos socio-espaciales en un escenario transformado es necesario conocer sus significados.

Los desastres socionaturales y las acciones de reconstrucción despliegan la activación de una conciencia del entorno construido y la circulación de narrativas por parte de las comunidades afectadas, que articulan distintos significados sobre el espacio

5 Esta denominación asume la inseparabilidad de la relación naturaleza-sociedad, por tanto postula que las amenazas naturales interactúan con grupos humanos expuestos y que esta exposición es diferenciada por elementos como estatus socioeconómico, género, etnia, edad, discapacidad, estatus de inmigración, cultura, relaciones de poder y sistemas político-económicos (Red Cross, 2014; Wisner, Blaikie, Cannon y Davis, 2003).

6 Berroeta, Ramírez, Ramoneda y Arrieta, s.f.

7 Low y Altman, 1992; Tuan, 1974.

8 Stedman, 2003. público; también se discute sobre el modo en que se quiere reconstruir, se relata la pérdida y el valor atribuido a lugares que ya no están, se lucha por participar de las decisiones constructivas y se busca reconstruir y mejorar la vida comunitaria. Estos relatos son asimilables a los grandes discursos del espacio público que operan como una suerte de dispositivos que significan el objeto y condicionan las prácticas de apropiación. Desde esta perspectiva, el sentido ideológico del espacio público se configura de manera distinta, en función de las redes de relaciones hegemónicas ${ }^{9}$ que lo producen, las que se materializan en las actividades que en él desarrollan, las formas físicas que adquiere y las regulaciones que se le imponen. En consecuencia, su significado es diverso y se configura a partir de discursos y prácticas con sentidos distintos, dependiendo de las expectativas que de él se tenga, de la producción del orden social al que se le asocie y de las condiciones de su transformación.

En consecuencia, en la valoración que se hace del espacio público resulta fundamental el rol que se

9 Žižek, 1994. 
le atribuye en la vida urbana. Según Rivlin ${ }^{10}$, se debería considerar la capacidad que tiene para representar a la diversidad de la sociedad de la cual es parte (edad, género, etnia, cultura), el grado de libertad de elección que permite en su uso, la coexistencia de actividades que facilita sin que se genere conflicto y las posibilidades que entrega para gestionar los conflictos armónicamente.

Desde este marco el planteamiento es que las necesidades y los requerimientos de los usuarios se transforman en los principales elementos de caracterización y evaluación del espacio público urbano ${ }^{11}$. Requerimientos que incluyen una variedad de demandas agrupables en necesidades asociadas a los usos específicos, a la construcción de significados espaciales y a las características físicas de los entornos.

En este texto presentamos parte de los resultados del proyecto Fondecyt "Vínculos socioespaciales en contextos de transformación urbana producida por catástrofes naturales", en el que nos propusimos explorar transversalmente la configuración de los significados en torno al espacio público en personas que han estado expuestas a procesos de transformación urbana producto de desastres socionaturales.

\footnotetext{
10 Rivlin, 1994.

11 Carr, Francis, Rivlin y Stone, 1992; Francis, 2003; Gehl, 2002 y 2014.
}

\section{Marcos discursivos del espacio público}

Es posible identificar tres grandes perspectivas o tendencias discursivas que se sitúan en la base de las políticas de representación del espacio público, a partir de la revisión de la literatura interdisciplinar sobre el tema. Estas tres perspectivas plantean tres visiones distintas sobre el pasado y el futuro de los espacios públicos en relación a los cambios contemporáneos en la vida pública, y sus presuposiciones y argumentos muestran sensibilidades analíticas dispares, con consecuencias políticas e ideológicas notablemente diferentes. Hemos llamado a estas tres perspectivas la tesis terminal o del espacio público perdido, la tesis optimista o la construcción de civilidad, y la tesis conflictivista o del control y disputa.

El espacio público perdido ${ }^{12}$ o el discurso terminal se refiere a la pérdida de un espacio público que fue compartido y democrático, denunciando la privatización y la homogeneización de la ciudad. En esta noción, el espacio público es disuelto para dar lugar a un espacio privado e individual, que

12 Berroeta y Vidal, 2012. 
obedece a diferentes modos de control, seguridad, sociabilidad y estética ${ }^{13}$. Esta noción de espacio público habla de un discurso terminal donde se sostiene un pasado idealizado sobre el lugar y se vislumbra en la contemporaneidad su fin. Esta representación terminal implica que el espacio público fue vivo y querido en un pasado. Aquí no se identifican modos de resistencia para evitar la pérdida del espacio, ignorando el conflicto social. Es una noción que se expresa con un tenor romántico e idealizado.

La comparación que hace Sorkin ${ }^{14}$ entre el espacio público actual y un parque temático sintetiza bien la tesis terminal. Este autor señala que el urbanismo actual se basa en la creación de espacios públicos que se caracterizan por no configurar un lugar, es decir, no son socialmente significativos, ni poseen una identidad de referencias locales; frecuentemente son simulaciones de espacio público antiguo que se estructuran desde una demanda por la seguridad y el control, que buscan asegurar que las personas no deseadas (porque no consumen o porque son socialmente discriminadas) no puedan entrar.

El espacio público como espacio de construcción de civilidad o el discurso optimista no niega la

13 Sennett, 1978.

14 Sorkin, 1992. noción anterior de espacio perdido, sin embargo, califica como inadecuada la visión romantizada del lugar pasado. Este discurso optimista considera que el espacio público debe adaptarse a las nuevas formas de transformación, trabajando para descubrir y proponer nuevos espacios públicos y modos de intervención para la calidad de vida. En esta noción, el espacio público es concebido como base para la realización de la ciudadanía, siendo un derecho humano fundamental ${ }^{15}$. Dentro de esta perspectiva se ubica la mayoría de las propuestas de criterios óptimos de intervención sobre el espacio público que teóricamente garantizan un buen funcionamiento social: derecho de presencia, uso y acción, apropiación, modificación y disposición ${ }^{16}$; posibilidades de comer, sentarse, relajarse, mirar y disfrutar ${ }^{17}$; fomento de una diversidad social; accesibilidad universal; participación en su creación, uso y gestión; control por parte del usuario; calidad ecológica; posibilidad de diversidad sensorial ${ }^{18}$, etc.

El espacio público como espacio de control y disputa o el discurso conflictivista postula una perspectiva crítica de la representación romántica de espacio público igualitario, identificando la disputa por el control del espacio y la oposición manifiesta en prácticas de apropiación del espacio. En

\footnotetext{
15 Berroeta y Vidal, 2012.

16 Lynch, 1984.

17 Whyte, 1980.

18 Abu-Ghazzeh, 1996; Appleyard, 1981; Francis, 1989.
} 
esta noción, el espacio público nunca ha sido ideal e igualitario, denunciando procesos de exclusión y segregación ${ }^{19}$. El espacio público aquí sólo se construye a través de la apropiación de los habitantes, material y simbólicamente en la lucha por el derecho a la ciudad, en procesos de una dialéctica dominación-contestación, poder y resistencia, orden transgresión, entre públicos y contra-públicos en situación de desigualdad ${ }^{20}$. Este discurso conflictivista denuncia las políticas de exclusión social y discriminación en la búsqueda por tener un lugar y hacérselo propio ${ }^{21}$.

De acuerdo con esta perspectiva, el derecho a la ciudad se basa en una distribución desigual de un derecho naturalizado a excluir ${ }^{22}$. Así, el espacio urbano sólo alcanza su estatus público cuando los sectores sociales más desfavorecidos, visualizados como excluibles por el ideario dominante, se apropian del territorio visibilizando sus reivindicaciones y necesidades, o cuando emergen usos espaciales espontáneos e imprevisibles ${ }^{23}$, no mediados por el Estado ni el mercado. En este proceso de conquista, el espacio no sólo es repensado simbólicamente sino que es producido materialmente pues deja de ser un escenario amplificador de la exclusión, la desigualdad, la resistencia política y

\footnotetext{
Berroeta y Vidal, 2012.

Di Masso, 2009.

Fraser, 1990; Mitchell, 1995.

Staeheli y Mitchell, 2008.

Cottino, 2005.
}

la imprevisibilidad urbana, para transformarse en un producto material de disputas por el derecho a la ciudad ${ }^{24}$.

Estos tres marcos discursivos resultan inconmensurables respecto a la esencia, configuración y proyección del espacio público actual y plantean sentidos y desafíos diversos a la ciudad del futuro. Por tanto, explorar su configuración en los relatos de personas que habitan entornos que se han modificado a consecuencia de desastres socionaturales, donde la conciencia y el deseo de proyectar espacios públicos es evidente, nos permiten evidenciar el entramado de representaciones variables y contestadas que articulan formas culturalmente estabilizadas de construir y regular el significado del espacio público. Movilizan simbólica y materialmente los mecanismos y fuerzas sociales que definen los contornos normativos de su significado y que legitiman las acciones materiales realizadas en su nombre.

24 Lefebvre, 1978; Mitchell, 2003. 


\section{Significados espaciales y espacio público}

Los espacios públicos significativos son aquellos donde las personas establecen una conexión entre su vida, tanto a nivel biográfico como cultural; se trata de un proceso interactivo que evoluciona en el tiempo y que afecta tanto a usuarios como a espacios ${ }^{25}$. Las características que adoptan estas conexiones varían, ya que pueden estar asociadas a experiencias pasadas o expectativas de acciones futuras, a la historia cultural de un determinado grupo, a contenidos de memoria individual y colectiva, o a características físicas o psicológicas. Es decir, el entorno urbano provee una serie de estimulaciones y las personas proveen su propia historia. Esta articulación es lo que conforma el significado en o del espacio público.

El modo en que se estructuran estos significados espaciales es explicado por Gustafson ${ }^{26}$ a partir del análisis de significados atribuidos al lugar de manera espontánea; este autor señala que los significados espaciales pueden distinguirse por características asociadas al sí mismo, a los otros o al propio ambiente.

25 Carr, Francis, Rivlin y Stone, 1992.

26 Gustafson, 2001.
Dos dimensiones fundamentales en la exploración de los significados son los aspectos socioculturales y la experiencia personal. El trasfondo sociocultural es fundamental para descubrir los tipos de lugares que son significativos, el rol que estos lugares cumplen y los procesos en los que se desarrollan los significados ${ }^{27}$, mientras que la experiencia personal es central en la atribución del significado espacial, pues las personas evalúan su relación con los lugares de la misma manera en que podrían evaluar relaciones con ciertas personas o ciertas actividades $^{28}$.

La relación que se produce entre los significados espaciales y la apropiación espacial, según Brower ${ }^{29}$, se basa en tres componentes que interactúan entre sí: la ocupación, manifestada a través de un conjunto de signos territoriales; la defensa del espacio, cuando se produce o se percibe una amenaza; y el apego que se tiene con un territorio producto de la identificación que se establece con él. La relación entre estos componentes se da mediante el apego y la identificación con el lugar, que responden a las cualidades simbólicas del propio lugar y a las acciones de defensa que se han llevado a cabo en él, las que, a su vez, producen y refuerzan el apego y la identificación, es decir, se trata de un proceso

\footnotetext{
27 Manzo, 2005.

28 Williams, 2008.

29 Brower, 1980.
} 
recursivo que $\mathrm{Pol}^{30}$ denomina "modelo dual de la apropiación espacial".

En consecuencia, los marcos discursivos del espacio público, la construcción de los significados espaciales y la apropiación espacial, son distinciones de un proceso recursivo que estructura y se estructura a partir de la representación normativa del espacio urbano, y se hace visible en forma de esquemas de premisas que fundamentan lo pensable y lo deseable, lo correcto y lo censurable, en relación al espacio público. Es así que los marcos discursivos se constituyen en el trasfondo ideológico normativo desde donde se configuran los significados en torno al espacio público y se fundamentan las acciones que en su nombre se desarrollan.

Las transformaciones producidas por desastres socionaturales afectan tanto la estructura como los significados espaciales que los individuos y las comunidades sustentan ${ }^{31}$. Los procesos de reconstrucción o relocalización intensifican cambios a nivel simbólico, pudiendo alterar los modos como una comunidad se organiza, se identifica y se apropia de un espacio.

Comunidades afectadas por desastres socionaturales ofrecen una mirada única para el estudio de la capacidad de resistencia y resiliencia ante una ruptura con el lugar ${ }^{32}$. Los procesos de reconstrucción

30 Pol, 1996.

31 Berroeta, Ramírez, Ramoneda y Arrieta, s.f.

32 Stedman, Amsden, Beckley y Tidball, 2014. se enlazan subjetivamente con prácticas para la restauración de los significados del espacio, asociándose con la revinculación con el lugar. Aquí, el habitar se enmarca desde una multidimensionalidad de significados, desde los elementos materiales del entorno, sus usos y sus transformaciones, en una fuerte relación entre habitar y construir. Procesos de reasentamiento de poblaciones vulnerables, por ejemplo, deberían realizarse construyendo conjuntamente un nuevo espacio en el cual se pueda significar, apropiar, arraigar y ser agente de su propio habitar ${ }^{33}$.

Con el objetivo de explorar la configuración de los significados en torno al espacio público en personas que han estado expuestas a procesos de transformación urbana producidos por desastres socionaturales, el presente estudio se realiza en relación a tres de los desastres socionaturales que ocurrieron en los últimos años en Chile y que resultaron en el desplazamiento o reconstrucción de barrios: (1) el terremoto del año 2007 en Tocopilla, en la zona norte del país; (2) la erupción del volcán Chaitén en 2008 en la ciudad de Chaitén, en la zona sur; (3) y el terremoto y tsunami de 2010, en la zona centro sur.

33 Chardon, 2010. 


\section{TOCOPILLA, 2007}

El terremoto de Tocopilla ocurrido el 14 de noviembre de 2007, generó la pérdida de la vivienda para aproximadamente 15 mil personas. Además, se produjeron deterioros en los sistemas de abastecimiento de agua, alcantarillado y en la red eléctrica. Se destruyeron vías de acceso, carreteras y calles, por lo que la ciudad quedó aislada. Ante esta situación, se realizó la reconstrucción de viviendas a los propietarios que fueron afectados de modo severo. Por otro lado, la solución para los allegados afectados por el terremoto, fue la planificación de tres proyectos de departamentos. A la espera de la entrega de las soluciones habitacionales se crearon 28 barrios transitorios donde convivían propietarios, allegados post-terremoto y allegados históricos que esperaban una solución habitacional hace más de una década. Una de estas soluciones fue el barrio Pacífico Sur. Localizado al sur de la ciudad de Tocopilla, fue entregado en noviembre de 2010 y abarca una superficie de 15 manzanas con un total de 324 casas, de las cuales 58 acogen a familias provenientes del sector Huellita, 30 de El Teniente y 236 viviendas fueron asignadas a allegados históricos y allegados post-terremoto. Las casas son pareadas, de madera tratada, con techo aislado de calaminas y de dos pisos, con una superficie promedio de terreno de 120 mts 2 y entre 53 a 56 mts 2 construidos, distribuidos en un primer piso con living comedor, cocina, baño y un pequeño patio que permite ampliación. Pacífico Sur no cuenta con espacios públicos, áreas verdes ni juegos infantiles, y existen seis sitios eriazos de distinto tamaño, algunos de los cuales muestran esbozos rudimentarios de plazoletas ${ }^{34}$.

\section{CHAITÉN, 2008}

El 2 de mayo de 2008, producto de la erupción del volcán Chaitén, fueron evacuadas 4.700 personas habitantes de la región. Anterior a la erupción, la comuna de Chaitén ya era una localidad de difícil acceso, situación que sólo disminuyó a partir de 1980 con la inauguración de la Carretera Austral y el consecuente desarrollo del turismo. La comuna identificada como la puerta de entrada de la Patagonia, se caracteriza demográficamente por una baja densidad poblacional, manteniendo una importante cantidad de terreno ocupada por bosques nativos. Tras la erupción volcánica, la mayoría de las viviendas fueron compradas por el Estado y se entregó un subsidio de subsistencia por 18 meses. Un número importante de habitantes se reasentó en distintas ciudades de la región de Los Lagos. Una de las localidades que recibió un número considerable de chaiteninos fue la comuna

34 Gobierno Regional de Antofagasta, 2008; Berroeta, Ramírez, Ramoneda y Arrieta, s.f. 
de Puerto Montt, específicamente la localidad de Alerce. Actualmente viven en Alerce cerca de 60 mil personas. El sector mantiene características propias del entorno rural, destacándose viviendas con vastos terrenos de vegetación y la ausencia de pavimentación ${ }^{35}$.

\section{DICHATO, 2010}

Ante el gran terremoto y tsunami del 27 de febrero de 2010, más del $80 \%$ de Dichato fue devastado. Los daños de la localidad provocaron que toda la población que vivía en la zona costera fuera erradicada temporalmente en Aldeas de Emergencias en zonas altas de seguridad. El plan de reconstrucción es llevado a cabo por distintos organismos públicos y técnicos, no obstante, el proceso fue lento. Los conjuntos habitacionales Villa Horizonte y Bahía Azul fueron construidos en el marco del proceso de reconstrucción. El conjunto habitacional Bahía Azul fue entregado en noviembre de 2012. Consta de 128 departamentos, cada uno con tres habitaciones dormitorios, un baño, una logia, un living-comedor, cocina y un balcón. Las áreas verdes y los espacios de circulación del conjunto habitacional Bahía Azul comprenden $9.109 \mathrm{~m}^{2}$. Este conjunto habitacional no cuenta con negocios en su interior, pero existe comercio cercano. Los residentes informan

35 Ministerio del Interior y Seguridad, 2014; Berroeta, Ramoneda y Opazo, 2015. que presenta problemas por falta de sistema de escurrimiento de aguas lluvias en los edificios, además de filtraciones y acumulación de agua. Los dirigentes también acusan que las áreas verdes no han sido gestionadas por la empresa constructora.

El conjunto habitacional Villa Horizonte fue entregado en febrero de 2013 y al igual que Bahía Azul cuenta con problemas de deslizamiento de barro producto de aguas lluvias. A estas problemáticas se suman algunas viviendas cuyos terrenos se están hundiendo, por lo que han tenido que ser reparadas. Villa Horizonte cuenta con 13 manzanas de viviendas edificadas. Sus espacios públicos se caracterizan por estar en proceso de construcción y/o abandono, a primera vista. Con respecto a sus organizaciones comunitarias, existe una junta de vecinos $^{36}$.

\section{CONSTITUCIÓN, 2010}

En el año 2010, Constitución también fue azotada por el terremoto y tsunami del 27 de febrero. Producto de este sismo, el conjunto habitacional Santa Aurora, emplazado en lo alto del cerro O'Higgins, fue demolido y reconstruido en el mismo emplazamiento. En 1998 se entregaron viviendas sociales a un grupo de pobladores, quedando consolidado el conjunto habitacional, que constaba de 84

36 Ministerio de Vivienda y Urbanismo, 2010 y 2013; Berroeta, Ramírez, Ramoneda y Arrieta, s.f. 
departamentos de 44,9 mts $^{2}$ ubicados en torres de tres niveles cada uno, sin espacios públicos disponibles para la comunidad. Sin embargo, post-terremoto y durante 3 años de reconstrucción, muchas familias tuvieron que vivir en precarias condiciones, percibiendo escaso apoyo de las autoridades. En 2013 se entregan los 48 departamentos de 55 mts2 para las 48 familias del comité habitacional Santa Aurora, los cuales se encuentran agrupados en tres blocks de 4 pisos cada uno, acogiendo a 149 personas aproximadamente. Actualmente cuenta con una junta de vecinos y un comité de co-propiedad. Posee espacios públicos que incluyen estacionamientos, un jardín, un quincho, áreas de juegos y una pequeña cancha de fútbol. Posee además un mini-market y acceso a la zona comercial de Constitución, ubicándose a 6 cuadras del centro cívico ${ }^{37}$.

\section{Metodología}

Desde una aproximación cualitativa, se realizaron como técnica de producción de datos, grupos focales, en las cuatro localidades. Para la selección de los barrios, los criterios fueron la existencia de algún grado de conocimiento previo entre los habitantes de los nuevos barrios, la antigüedad del barrio y que la tipología de las viviendas fuera

37 Ministerio de Vivienda y Urbanismo, 2010; Berroeta, Ramoneda y Opazo, 2015. similar. De esta forma, para el caso del terremoto de Tocopilla de 2007, entre los dos barrios más antiguos que se construyeron se seleccionó el barrio Pacífico Sur, que tiene un sector de vecinos que fue reubicado en su conjunto. En el caso del terremoto del año 2010, considerando que la asignación de viviendas fue en base a criterios individuales, en Constitución se seleccionó el conjunto habitacional Santa Aurora por ser un barrio que fue completamente demolido y reconstruido manteniendo sus mismos habitantes. En Dichato se seleccionaron los barrios Villa Horizonte y Bahía Azul, que fueron habitados por personas que residían en sectores muy cercanos. Para el caso de Chaitén, como no se generó un proceso de reconstrucción propiamente tal y lo que se hizo fue entregar un subsidio individual, se pesquisaron distintos asentamientos donde se reubicaron los habitantes, seleccionando la localidad de Alerce en Puerto Montt, donde vive un grupo aproximado de 150 familias.

Durante el año 2014 se realizaron 17 grupos focales, con un total de 117 participantes, formados por cuatro grupos con personas desplazadas de Chaitén, tres en Tocopilla, seis en Dichato y cuatro en Constitución.

Los participantes componen una muestra de tipo intencional y los criterios de selección fueron: personas mayores de 18 años de ambos sexos, 
propietarios de sus viviendas, que hayan residido por más de cinco años en el barrio de origen de la comunidad y que no hayan sufridos daños físicos ni pérdida de familiares o amigos como consecuencia de los desastres. Si bien esta investigación no analiza los discursos de género, se asume su importancia en la construcción de los significados del espacio público. Por lo tanto, se opta por presentar, luego de cada viñeta, si ésta es construida por una mujer o por un hombre, de modo de ayudar al lector a una comprensión más situada del discurso.

La selección de los participantes se realizó a partir de quienes señalaron su voluntad durante la aplicación de cuestionarios en una fase previa de la investigación y de la intermediación de informantes claves de cada comunidad. Los procedimientos éticos de investigación se efectuaron mediante las firma de consentimientos informados. Los grupos focales fueron grabados y transcritos. La información fue organizada utilizando el software ATLAS.ti 7. El método de análisis se inspira en la grounded theory ${ }^{38}$. Se hicieron procedimientos de codificación, generación de categorías e identificación de relaciones entre las categorías emergentes. Se realizó un análisis abierto inicial y un análisis axial de los datos. El análisis siguió una lógica individual analizando cada localidad por separado,

38 Strauss y Corbin, 2002. y luego una lógica transversal desde los ejes temáticos-analíticos emergentes en todos los $\operatorname{casos}^{39}$. El proceso de categorización global fue orientado teóricamente desde los marcos discursivos identificados para los relatos del espacio público. Conceptualmente, estos discursos engloban distintos repertorios interpretativos que definen los significados asociados al espacio público.

\section{Resultados}

A partir de los marcos discursivos revisados, se identificaron categorías emergentes y transversales en los casos estudiados que serán descritas a continuación. Estas categorías se organizan del siguiente modo: (1) discursos de la pérdida: (a) arraigo y pérdida de modos de vida; (b) añoranza del espacio perdido; y (c) privatización del espacio público. (2) Discursos de la civilidad: (a) la comunidad ideal es la que está unida; (b) prácticas culturales y vecinales; y (c) prácticas de cuidado con el espacio público. (3) Discursos del espacio como control y disputa: (a) protestas como herramienta de conquista del espacio; (b) abandono y estigma; y (c) participación para la garantía de derechos.

39 Cornejo, Besoaín y Mendoza, 2011. 


\section{Discursos de la pérdida}

Los relatos de la experiencia de las transformaciones por un desastre socionatural explicitan la pérdida de elementos tanto individuales como comunitarios que convergen en un discurso pesimista. En su conjunto relatan una localidad que ya no es la misma después del evento, en dimensiones físicas y relacionales. Se observa que para describir la pérdida, la base de los relatos es la presencia previa de un arraigo con el entorno que se desarrolla en valoraciones positivas hacia el espacio pasado, identificando lo que fue perdido y su consecuente mal-estar y melancolía actual. En esta posición se dificulta la apropiación hacia el nuevo espacio, así como el consecuente vínculo. Este lugar perdido mantiene el recuerdo de las funciones de protección, familiaridad y acogida de los participantes ${ }^{40}$.

Está presente también la percepción de privatización de los espacios, incluyendo la búsqueda por seguridad individual y la pérdida de espacios de socialización. Los modos de vida vinculados al espacio perdido son valorados como ideales, y los cambios tras la pérdida revelan habitantes que ya no se hallan en una comunidad. Rescatando a Davis $^{41}$, esta búsqueda por seguridad en los nuevos espacios públicos demuestra un desplazamiento

40 Bachelard, 2000; Besoaín, Morales, Soto, Hidalgo, Fernández, Bernal y Carvalho, 2015. .

41 Davis, 2002. del público hacia lo privado, emergiendo un protagonismo del individual y un sentimiento de amenaza por la presencia del otro. Esta búsqueda por la seguridad lleva a una "destrucción de cualquier espacio público auténticamente democrático"*2. Esta tendencia se asemeja a las reflexiones de Atkinson y Flint ${ }^{43}$ sobre la sustitución de los barrios por comunidades cerradas y las críticas de Smith ${ }^{44}$ al "urbanismo neoliberal".

\section{ARRAIGO Y PÉRDIDA DE MODOS DE VIDA}

El lugar anterior a las transformaciones es relatado desde las diferencias comparativas con la vida actual, puntuando la relación entre espacios y momentos significativos de la vida comunitaria -negativos o positivos- que adquieren un sentido de cohesión, valoración y pertenencia al lugar, valores que son sustentados desde una visión bucólica de una comunidad ideal que se apropiaba de los espacios públicos.

Sí, teníamos nuestro espacio, la sede, nuestro grupo folclórico, bailábamos, teníamos cancha. Teníamos buenas relaciones con los vecinos, hacíamos actividades, carnavales, ganamos proyectos, podía bajar a pie al centro si quería, ahora no, tengo que tomar locomoción sí o sí. Por eso hay diferencias. Yo acá tengo casa propia pero a la vez no tengo nada, no

\footnotetext{
42 Ibíd, p. 178.

43 Atkinson y Flint, 2004

44 Smith, 2005.
} 
participo en nada, sólo a las reuniones, no tengo espacio en comunidad con mis vecinos, son los puntos que tenía antes y que no los tengo ahora. (Mujer, Pacífico Sur, Tocopilla).

Considerando las diferentes trayectorias de las transformaciones y los procesos de reconstrucción, los relatos adquieren distintas valoraciones del espacio perdido. En el caso de Chaitén, los desplazamientos a localidades lejanas en cuyas comunidades se despliegan formas de vida distintas a las de Chaitén, como es el caso de los desplazados en Puerto Montt, se reconocen a través de modos de vida que ya no ocurren. La imposibilidad de realizar determinadas prácticas sociales y el desagrado por las nuevas formas de movilidad urbana en el territorio denuncian las diferencias del ritmo de vida entre un antes perdido y un después incómodo. Los males de la ciudad grande y su individualismo parecen metaforizar el no hallarse de una población arraigada a un lugar con características rurales.

Igual era mejor que acá. La gente que salió de allá ahora vive cada uno en su lugar, ya se perdió eso que cuando uno se encontraba se saludaba con cariño, se conversaba, ahora ya no pasa. La gente cambió, es un cambio grande, como que se habitúa a la costumbre que hay acá cada uno no le interesa lo que pase con el resto, su vecino que está al lado, nada. Allá era distinto había comunicación, se visitaban los vecinos, ¿cómo está vecino? Acá no, yo echo de menos mucho eso. Cuando llego allá todos me saludan porque todos me conocen, conversamos. Acá todos andan apurados, yo vengo almorzar de allá y tengo dos horas, pero si pago locomoción es plata que se va y si camino me demoro $20 \mathrm{mi}-$ nutos para venir y 20 para volver, tengo una hora para comer apuradito, es complicado. (hombre, Los Alerces, Chaitén).

A diferencia de Chaitén, la trayectoria de reconstrucción de Dichato permitió que los habitantes identifiquen la pérdida desde la re-significación positiva. Sin embargo, no deja de estar presente la visión romántica del espacio público. Se valoran positivamente las mejorías de las condiciones habitacionales y la mayor visibilidad nacional que obtuvo la ciudad, pero se manifiesta en paralelo una pérdida de modos de vida, mediante la metáfora de un pueblo que se "civiliza" y adquiere los males de la ciudad grande.

La mayoría salió de un Dichato antiguo, un Dichato de tabla porque mayormente era todo de tabla y adobe que muy poco se afirmaron y salieron borradas del mapa porque no eran construcciones sólidas. De la noche a la mañana empieza a desaparecer este Dichato y cambió el modo de pensar de la gente, que se civilizó, empezó de a poquito darse cuenta y empezó asimilar el buen vivir, a asomarse, pero ha cambiado, el ruido ya les molesta, el mal de vivir de otros lo están mirando, antes no había que hacerse el leso no más. (hombre, Bahía Azul, Dichato). 


\section{AÑORANZA DEL ESPACIO PERDIDO}

Dentro de los discursos de la pérdida se inscribe la añoranza de un espacio portador de cualidades promotoras de la sociabilidad. Este sentimiento se manifiesta justificado principalmente por las consecuencias provocadas por el diseño y emplazamiento de las nuevas viviendas, que son expresadas como una disminución de la seguridad, privacidad y conectividad en el territorio.

Una casa que sea no pareada tienes más espacio, más libertad con los niños. Aquí en un departamento no hay eso. Aquí en el edificio son 4 departamentos uno sube por la escalera se escucha por toda la torre. No puedes ni siquiera tener una conversación privada, tiene que ser como al oído, porque se escucha todo. (hombre, Bahía Azul, Dichato).

Extraño la tranquilidad, estar más cerca de las cosas, las facilidades como la cancha, el centro, la misma escuela estábamos a pasos de la escuela. Los mismos niños que viven aquí, yo creo que igual pa' bajar a la escuela y llegar allá igual les cuesta. Se pegan las medias caminatas. (hombre, Villa Horizonte, Dichato).

En el caso Chaitén y Tocopilla, algunos habitantes expresan también la idea de la pérdida de una comunidad cerrada, señalando que las transformaciones, principalmente la llegada de nuevos habitantes, disminuyen las características de pertenencia a su pueblo.
Todavía no está el "Chaitén antiguo"... Todavía no lo limpian bien. Es que se ha ido tanta gente que... Sí, otra gente ya no va a ser el mismo Chaitén que era... Mucha gente de afuera (mujer, Los Alerces, Chaitén).

El barrio donde yo vivía ahora viven puros colombianos casi. No se veía mucho la raza colombiana acá, pero ahora está lleno, están arrendando y viven bolivianos y peruanos" (mujer, Pacífico Sur, Tocopilla).

\section{PRIVATIZACIÓN DE LOS ESPACIOS}

Los habitantes aseveran que la relación con sus vecinos se transformó. Después de los desastres socionaturales emergieron actitudes más individualistas y defensivas por parte de los vecinos. Se describe la privatización de los espacios públicos y la ausencia de espacios de recreación y vida comunitaria. Esta privatización del espacio público se produce principalmente por problemas de convivencia con los vecinos actuales, en lo que se refiere a la privacidad y al poco conocimiento mutuo.

Producto de todo lo que pasó, la gente que antes era súper alegre, en ese momento como que... cambió todo, considerablemente, en el sentido de que antes uno podía ayudar, ayudaba, ahora hay gente que no poh, que no ayuda a nadie. Y aunque tenga la posibilidad de ayudarte, ellos no lo hacen. Será por lo que perdieron, también, cambiaron la mentalidad y dijeron "ya, yo tengo que ver por mí porque no 
es el momento...". Uno se va por eso. Les cambió el switch no más y dijeron: "pucha, yo ya ví por los demás, ahora veo por mí (mujer, Villa Horizonte, Dichato).

\section{Discursos de la civilidad}

El discurso optimista del espacio público transformado por un desastre socionatural concibe de forma importante la adaptación y las prácticas de intervención para mejorar la calidad de vida de los habitantes. También está presente la noción de pérdida, sin embargo, en estos relatos la pérdida no se significa como un discurso pesimista, sino como una motivación para la acción y la reconstrucción.

A diferencia del discurso anterior, aquí los habitantes proponen y realizan acciones para la mejora de los espacios, trabajando para garantizar viviendas y espacios públicos adecuados. Es un trabajo primordialmente comunitario que construye espacios que se pueden apropiar $y$, de cierto modo, controlar.

Respecto al proceso de transformación de los espacios, el relato de los habitantes se despliega desde la resignación, concibiendo sus prácticas como modos de reapropiación, preocupación y cuidado de los espacios públicos. Estas prácticas se alinean a la descripción de Borja ${ }^{45}$, que considera que nos encontramos "ante el desafío de hacer ciudad; ejercer el derecho a la ciudad por parte de los ciudadanos es lo que hace a la ciudad viva en el presente, capaz de reconstruir pasados integradores y proponer proyectos de futuro movilizadores"46. Desde esta posición el espacio público opera como objeto de finalidad para alcanzar una mejor calidad de vida, a la vez que se transforma en un promotor de la conciencia ciudadana que ejerce su derecho a intervenir el entorno.

\section{LA COMUNIDAD IDEAL ES LA QUE ESTÁ UNIDA}

Las transformaciones del espacio son vividas desde una aceptación resignada, enfatizando los aspectos positivos, incluso cuando la situación en que se encuentran no cumple con sus expectativas. A partir de ello, los participantes cultivan la idea positiva de que una comunidad ideal es aquella que está unida y con participación activa de los vecinos. Identifican que es importante que las personas se conozcan, compartan, se apoyen, pero sobre todo que construyan conjuntamente un sentimiento de seguridad en la comunidad. La unión es entendida también como facilitadora para afrontar las dificultades que se generan en el proceso de reconstrucción. 
En el caso de Constitución, la unión es descrita como el motivo por el cual se conquistó la reconstrucción del barrio. En un proceso recursivo, las reuniones constantes en torno a este objetivo común son descritas como potenciadoras de la unión.

Lo principal que ellos estén unidos, que todos remen pal mismo lado, yo creo que la unión hace la fuerza y cuidar su espacio, porque aquí llegaba gente que venía a robar! Y le decían joh vecina, ¿así que vino a buscar su baño? No! ¡Y como yo vi que lo vino a buscar! Yo creo que pal terremoto salió todo lo malo del ser humano, lo mismo que está pasando en Iquique, que están vendiendo el pan a $\$ 1000$, entonces esas cosas malas sobresalen! (mujer, Santa Aurora, Constitución).

\section{PRÁCTICAS CULTURALES Y VECINALES}

La interacción entre vecinos que se desarrolla en torno al espacio público es significada como una acción ciudadana que se construye y se organiza para apropiarse del entorno transformado, inclusive en condiciones de precariedad material. Se describen prácticas culturales como teatro callejero, talleres de costura, cuentos y carnaval que son desarrolladas a pesar de la falta de espacios comunes.

Se hacen talleres de costura, de tejido, para eso falta un lugar, porque la sede no sirve como un lugar para taller, la verdad no sirve, si hay reunión u otra actividad los talleres no pueden funcionar (mujer, Villa Horizonte, Dichato).
Como modos de organización vecinal se destacan las juntas y los comités de vecinos, que en los relatos son valorados como facilitadores de apoyo económico y emocional para la comunidad. Se destacan espacios de confianza como el almacén del barrio y las reuniones de mujeres, a la vez que se realizan acciones de cuidado y vigilancia para proteger espacios comunes. Aquí, la ciudadanía es concebida y valorada como un repertorio colectivo de confianza y colaboración.

Siempre cuando a alguien le pasa algo, todos estamos pendientes. Yo no estoy siempre, pero si tocan la puerta y necesitan algo, ahí está uno. En el block de nosotros nos juntamos a tomar once, para las reuniones... no tenemos sede todavía, están tratando de hacer una... (mujer, Santa Autora, Constitución).

\section{PRÁCTICAS PARA LA MEJORÍA DE LOS ESPACIOS}

La creación y mejoramiento de los espacios públicos es descrita como un derecho. Está presente la organización, el deseo y la realización de acciones para mejorar la infraestructura de los espacios públicos. Las iniciativas se refieren a acciones de limpiar y decorar sectores con plantas y árboles, construir plazas y multicanchas, instalar bancas, paneles solares y juegos infantiles. Estas iniciativas denuncian la falta de espacios públicos y la valoración de estrategias de autogestión para la reapropiación del espacio público, realizando inversiones 
de materiales y de tiempo. Aunque el discurso valore prácticas comunitarias, en los relatos aparecen innumerables dificultades del proceso de organización.

No, ahora se ven familias, lo que pasa que acá no hay espacios públicos y eso es lo que nosotros estamos peleando, imagínate que la sede está en un peladero para atrás, no hay nada (...) Los vecinos empezaron, lo que pasa que no había una capacitación que ahora queremos, como plantar un arbolito, porque hay gente que hicieron un hoyito y plantaron y listo, en una semana se murió el arbolito y la gente trataba de revivirlo pero las hojitas se caían. Ahora estoy en un procedimiento para que vengan los de la municipalidad y planten arbolitos ahí, decirles a los vecinos que cuiden su arbolito (mujer, Pacífico Sur, Tocopilla).

Porque también es cierto que la primera misión es entregar la casa, pero eso va comprometido con muchas otras cosas para realmente entregar una reconstrucción. Porque la reconstrucción no es vivienda no más. Hay que reconstruir familias, hay que reconstruir confianza, fuentes laborales, un montón de cosas, áreas verdes... (mujer, Villa Horizonte, Dichato).

\section{Discursos del espacio como control y disputa}

Las transformaciones producidas por desastres socionaturales provocaron la conciencia en los habitantes de que el espacio no es controlado libremente por ellos, sino que se trata de un espacio que se disputa con el Estado e instituciones privadas $^{47}$. Los participantes enfrentan esta disputa con organización comunitaria, respondiendo a la falta de control con luchas por la participación en la planificación y toma de decisión sobre las características que adoptarán el espacio público y las viviendas.

Está presente la percepción de injusticia y abandono por parte del Estado y la emergencia de estigmas peyorativos hacia los habitantes de localidades afectadas. Siguiendo a Burte ${ }^{48}$, el espacio público funciona como objeto, escenario y precipitador de conflictos.

En esta misma dirección se identifica la aparición de sujetos políticos entre los habitantes que disputan simbólica y materialmente la legitimidad de los espacios. Coherente a lo descrito por Ugarte y Salgado $^{49}$, "el territorio deja de ser sólo un escenario de acción para convertirse en elemento en disputa

\footnotetext{
47 Harvey, 2013.

48 Burte, 2003.

49 Ugarte y Salgado, 2014.
} 
y cuya soberanía se disputa contra el Estado"50 Así, además de precipitar conflictos, el espacio en este caso precipita el surgimiento de una consciencia de dominación y lucha. Se reconocen dos momentos. Inicialmente, los habitantes se encuentran en una posición pasiva, receptora de las decisiones gubernamentales. Esta posición luego es sustituida por la consciencia de la necesidad de una organización colectiva que dispute con el Estado los elementos del proceso de reconstrucción. Esta segunda etapa es dinámica: los habitantes inicialmente luchan realizando protestas, estrategia que es seguida por acciones colaborativas de negociación en los procesos de diseño y construcción de los espacios.

\section{PROTESTAS COMO HERRAMIENTA DE CONOUISTA DEL ESPACIO PÚBLICO}

Los habitantes relatan las acciones de protesta como una estrategia colectiva para visibilizar necesidades, preocupaciones e injusticias asociadas a la reconstrucción. Las protestas son significadas como propulsoras de la reconstrucción, propiciando que el gobierno se responsabilizara no solamente por las soluciones habitacionales, sino que también por más calidad y opciones de vivienda. A pesar de que algunos habitantes valoran negativamente las protestas, el discurso en su favor entiende que se justifican en contextos de lucha e injusticia, siendo una herramienta válida para visibilizar y conquistar su territorio.

La protesta fue decisiva para obtener las casas. Si Dichato está como está es fruto de la protesta, porque ahí nos sentamos a negociar, creamos mesas sociales, creamos la pega pal delegado presidencial, porque ahí salió el "Viva Dichato", la recuperación económica del pueblo... (mujer, Villa Horizonte, Dichato).

Nosotros tuvimos que pelearla, porque no querían ni construirnos, querían botarnos a todos pa' afuera, por eso tuvimos que tomarnos la carretera, después nos tomamos el SERVIU, fue una lucha (hombre, Santa Aurora, Constitución).

Por otro lado, frente a la imposibilidad de acción colectiva, como en el caso de los participantes de Chaitén y a diferencia de Constitución, Dichato y Tocopilla, la protesta es una práctica de reclamo individual ante las instituciones, que es señalada como ineficaz.

\section{PARTICIPACIÓN PARA LA GARANTÍA DE DERECHOS}

El control sobre el espacio público y la vivienda se muestra limitado por los habitantes. Las constructoras y el Estado detentan el control de los espacios, disminuyendo las posibilidades de transformación individual y comunitaria. Luego de las protestas, el proceso cambia ya que se desarrollan estrategias

50 Ibíd., p. 66. 
armónicas de negociación y se participa en alguna de las decisiones del proceso, pasando de la confrontación a la colaboración.

A mí el terremoto me mató, a mí no me entregó ni me dio nada bueno, sino puras cosas malas: me cambió de barrio, me sacaron de mi casa, estuve presa, me desalojaron con carabineros, me llegó una orden que tenía que desalojar la casa y ahí me tiraron a un campamento, donde hice un escándalo para que me dieran una media agua, me tuve que traer 6 media aguas. Yo tenía un palacio en el campamento. (mujer, Pacífico Sur, Tocopilla).

Como modo de contestación y dominación del control, los involucrados presentan como solución posible la participación en el proceso de reconstrucción de las viviendas y barrios. Esta participación se realizó tanto de manera individual como colectiva a través de los comités y directivas que se generaron para facilitar el proceso de obtención de los subsidios. La participación representa la lucha por el derecho al espacio, la visibilización de sus demandas y la fiscalización de que se garanticen sus derechos. La importancia de garantizar seguridad en la construcción de la vivienda es aquí un modo de evitar que se repita lo que sufrieron -pérdidas materiales y subjetivas- en el desastre.

Se destacan los casos de Villa Horizonte en Dichato, donde los participantes pudieron elegir el tipo, el material y el color de sus viviendas, así como los nombres de las calles de la villa. En Constitución, la participación se realizó mediante una serie de reuniones con los encargados de la reconstrucción, siendo partícipes del diseño de sus viviendas y la distribución de los departamentos, manteniendo, en la medida de lo posible, la distribución anterior al desastre socionatural.

Nos mostraron el plano, pero no nos gustó porque eran de 6 pisos y nosotros no queríamos de 6 porque se podían caer, teníamos miedo... porque se habían caído de 5 pisos, imagínese de 6 , entonces nos bajaron a 4 pisos (mujer, Santa Aurora, Constitución).

En Chaitén, a diferencia de Constitución, Dichato y Tocopilla, no hubo participación para la reconstrucción, ya que las personas fueron desplazadas a una nueva localidad. En este caso, la estrategia fue individual. Mediante un bono en dinero entregado por el gobierno, los habitantes buscaron el tipo de vivienda y el lugar donde comprar. La elección individual se orientó por el emplazamiento del barrio, teniendo en cuenta factores de seguridad y cercanía con familiares o vecinos de origen.

\section{LEGITIMIDAD Y ESTIGMA}

En el período de la emergencia, hasta la entrega de las soluciones habitacionales, los afectados, en su mayoría, fueron enviados a vivir por largos períodos a campamentos, aldeas, albergues y otras, en condiciones tan precarias material y emocionalmente que los participantes significan desde una sensación de abandono e injusticia. Aquí está presente una visión inicial del Estado como el 
controlador del espacio, y de los habitantes, como sujetos pasivos que reciben soluciones. Esta primera significación es relatada como el antecedente para la toma de conciencia de la necesidad de una organización colectiva que dispute con el Estado los elementos del proceso de reconstrucción.

No teníamos dónde estar, dónde vivir, así que cada uno busco dónde vivir por ahí, hubo mucha gente que anduvo a maltraer muchos días, y bueno, después hubo un largo periodo en que estuvimos viviendo en media aguas, otros de allegados con los familiares, los que podían arrendaban, pero sus muy pocos porque la situación era mala. La mayoría la sufrió. Después vino el periodo que nos tocó luchar para que nos reconstruyeran, que nos costó harto, costó peleas con las autoridades y discusiones por aquí y por allá. Venían las autoridades, nos visitaban, pero nunca se hacían las cosas inmediatamente como se hubiera querido (hombre, Santa Aurora, Constitución).

Las transformaciones del espacio generaron también estigmas que evidencian la segregación de determinados grupos. Los habitantes que fueron objeto de estas soluciones despiertan un sentimiento de estigmatización desde grupos o personas que no son parte de la comunidad afectada. En Bahía Azul, por ejemplo, se les nombra "Colina 2" por parecerse a módulos de cárcel. En el mismo sentido se expresan prejuicios o estereotipos asociados al lugar de origen de los desplazados, como en el caso de Chaitén. Se evidencia en ello una disputa simbólica por la legitimidad de los usuarios del espacio por haber sido beneficiados por la reconstrucción.

Fui a hablar con la asistente social y isabe lo que me dice? Me dice "sí poh, si la gente de Chaitén es de campo" Ellos tienen una imagen de Chaitén que la gente son todos campesinos, son todos ignorantes. Y yo le dije: "disculpe, pero yo no soy campesina, yo fui desplazada de Chaitén y tengo mi educación, este es mi segundo título, ya tengo mi título". Ése es el mal concepto que tienen ustedes, porque piensan que nosotros somos campesinos y que con decir un par de palabras nos van a tapar la boca y no nos ayudan absolutamente en nada (mujer, Los Alerces, Chaitén).

En suma, identificamos que los tres grandes marcos discursivos del espacio público -perdido, cívico y en disputa- se organizan en torno a: (a) la pérdida de elementos tanto individuales como comunitarios que convergen en un discurso pesimista; (b) la adaptación y las prácticas de intervención para mejorar la calidad de vida de los habitantes; y (c) la noción de que el espacio no es controlado libremente por sus habitantes, sino que es un espacio de disputa con el Estado e instituciones privadas. Este entramado de representaciones variables y contestadas articula formas culturalmente estabilizadas de construir y regular el significado del espacio público y moviliza simbólica y materialmente las estrategias y las fuerzas sociales que definen los contornos normativos de lo que es 
deseable o no y legitiman las acciones materiales realizadas en su nombre.

\section{Conclusiones}

La creación de espacio público urbano se puede producir típicamente por regeneración de espacios obsoletos no destinados originalmente a un uso público, por refuncionalización de espacios públicos ya existentes o por creación ex novo ${ }^{51}$. En cualquiera de los tres casos, la apertura de un espacio físico abierto en un entorno urbanizado activa y canaliza las dinámicas propias de la vida pública que caracterizan el medio sociocultural y político en el que se construye dicho espacio.

En los casos que hemos abordado, la transformación del entorno derivada de un desastre socionatural generó oportunidades de creación y restauración de espacio público que siguieron lógicas de resignificación espacial interpretables en clave local. No obstante, aun cuando cada localidad afectada produjo relatos contextualizados sobre la naturaleza, la función y el sentido del espacio público, de acuerdo con las características específicas del proceso de pérdida y restauración del lugar concreto, identificamos cómo los tres grandes marcos discursivos que rigen la producción de significados del espacio público se despliegan de un modo común. Es así que registramos la coexistencia de relatos del espacio público que se asocian al paradigma territorial de la civilidad y del consenso, o bien a la nostalgia romántica de un espacio idílico del pasado, pero también como un territorio de contestación y disputa para la consecución del derecho a la ciudad.

Los relatos operan como fundamento y justificación de las acciones individuales y colectivas que los habitantes de estas localidades emprenden. Estos discursos tienen un valor retórico e ideológico en el marco de procesos políticos y sociales más amplios de (des)legitimación de las estrategias de regeneración del entorno específicas de cada caso. Esta afirmación implica que lejos de tener un valor descriptivo, en el contexto de los procesos de duelo y reconstrucción socio-territorial derivados de un desastre socionatural, estos tres discursos sobre el espacio público funcionan social e institucionalmente como un recurso más de las comunidades afectadas y los poderes públicos para justificar o contestar modos de producción urbana en último término debatibles desde el punto de vista de la calidad de vida, la cohesión social, la justicia y la equidad.

El reconocimiento de cómo se materializan estos discursos nos permite develar las contradicciones y tensiones de los procesos de reconstrucción,

51 Borja y Muxí, 2003. 
evidenciar las paradojas que se generan entre las acciones desplegadas y los sentidos declarados, comprender los procesos de contestación y de lucha por la participación en la planificación y toma de decisión que demandan las comunidades, reconocer la producción de exclusión y estigma que las soluciones constructivas generan, y por sobre todo, des-construir el relato hegemónico de procesos naturalizados y armónicos que añora la estandarización de soluciones y estrategias que minimizan el trasfondo situado espacial y temporalmente de cada desastre socionatural.

Podemos afirmar entonces que la reconstrucción es un acto político ${ }^{52}$ donde los espacios están en disputa entre los intereses de los diferentes actores involucrados, en especial, del Estado, de las instituciones privadas y de las comunidades. Ahora bien, más allá de reconocer estas tensiones y dinámicas de contestación de las experiencias estudiadas, como hemos identificado en otros trabajos $^{53}$, y considerando la mirada tecnocrática del Estado chileno hacia el territorio y la gestión del riesgo, insistimos en la necesidad de que las estrategias que despliegan los gobiernos de turno deben valorar al capital comunitario y cultural de las regiones, a la vez que respeten los derechos que tienen las comunidades en toda intervención ${ }^{54}$.

52 Salgado, 2014.

53 Cox y Perry, 2011; Di Masso, 2015; Larenas, Salgado y Fuster, 2015; Red Cross, 2014; Salgado, 2014; Tapia, 2015.

54 Winkler, Reyes, Olivares y Berroeta, 2016.
Destacamos dos procesos que observamos en este estudio y que marcan una diferencia importante en la vinculación espacial de las comunidades: primero, que la relocalización en zonas geográficas diferentes es doblemente traumática si no se cuidan aspectos psicosociales medulares y, segundo, que las prácticas de participación de las personas desplazadas en la toma de decisiones con respecto a su relocalización resultan fundamentales para el proceso de apropiación y vinculación al nuevo entorno.

\section{Bibliografía}

ABU-GHAZZEH, Tawfiq. Reclaiming public space: The ecology of neighborhood open spaces in the town of Abu-Nuseir, Jordan. Landscape and Urban Planning. 36(3): 197-216, noviembre 1996. DOI 10.1016/S0169-2046(96)00343-X.

APPLEYARD, Donald. Livable streets. Berkeley, Estados Unidos, University of California Press. 1981. 382 p. ISBN 978-0520047693.

ATKINSON, Rowland y FLINT, John. Fortress UK? Gated Communities, the spatial revolt of the elites and time-space trajectories of segregation. Housing Studies. 19(6): 875-892, enero 2004. DOI 10.1080/0267303042000293982. 
BACHELARD, Gastón. La poética del espacio. México, Fondo de Cultura Económica. 2000. ISBN 950-557-354-5.

BESOAÍN, Carolina; MORALES, Rodrigo; SOTO, Alejandro; HIDALGO, Karla; FERNANDEZ, Ismael; BERNAL, Vicente y CARVALHO, Laís. El retorno al campamento: subjetivación urbana y sus resistencias en los márgenes de la ciudad formal. En: CONFLICTOS urbanos y territoriales: ¿desafiando la cohesión social? Conferencia Anual COES. Noviembre 2015. P. 1068-1100.

BERROETA, Héctor; RAMÍREZ, Catalina; RAMONEDA, Álvaro y ARRIETA, Carlos. Vínculos desplazados por desastres en Chile. En: OLIVOS, Pablo y NAVARRO, Oscar. Catástrofes: riesgo e identidad. S.f. En prensa.

BERROETA, Héctor; RAMONEDA, Álvaro y OPAZO, Luis. Sentido de comunidad, participación y apego al lugar en comunidades desplazadas y no desplazadas post desastres: Chaitén y Constitución. Universitas Psychologica. 14(4): 15-27, octubre 2015. DOI 10.11144/Javeriana.upl4-4.scpa.

BERROETA, Héctor y VIDAL, Tomeu. La noción de espacio público y la configuración de la ciudad: fundamentos para los relatos de pérdida, civilidad y disputa. Polis. 11(31): 57-80, abril 2012. DOI 10.4067/S0718-65682012000100004.

BORJA, Jordi. La ciudad conquistada. Madrid, Alianza. 2003. 381 p. ISBN 9788420641775.

BORJA, Jordi y MUXÍ, Zaida. Espacio público: ciudad y ciudadanía. Barcelona, Electa. 2003. ISBN 84-8156-343-9.
BROWER, Sidney. Territory in urban setting. En: ALTMAN, Irvin, ed. y WOHLWILL, Joachim, ed. Culture and environment. New York, Wiley. 1980. P. 179-207. Human Behavior and Environment, v. 4. ISBN 978-1-4899-0451-5.

BURTE, Himanshu. The space of challenge: Reflections upon the relationship between public space and social conflict in contemporary Mumbai. [En línea]. Centre de Cultura Contemporània de Barcelona. 2003. Disponible en: http://www.publicspace.org/ en/text-library/eng/a014-the-space-of-challengereflections-upon-the-relationship-between-public-space-and-social-conflict-in-contemporarymumbai.

CARR, Stephen; FRANCIS, Mark; RIVLIN, Leanne y STONE, Andrew. Public space. Cambridge, Cambridge University Press. 1992. 420 p. ISBN 978-0521359603.

CHARDON, Anne-Catherine. Reasentar un hábitat vulnerable. Teoría versus praxis. Revista INVI. 25(70): 17-75, noviembre 2010. ISSN 0718-8358. DOI 10.4067/S0718-83582010000300002.

CORNEJO, Marcela; BESOAÍN, Carolina y MENDOZA, Francisca. Desafíos de la generación de conocimiento en la investigación social cualitativa contemporánea. Forum Qualitative Sozialforschung / Forum: Qualitative Social Research. 12(1), enero 2011. ISSN 1438-5627.

COTTINO, Paolo. La ciudad imprevista. Barcelona, España, Bellaterra. 2005. 160 p. ISBN 9788472903005. 
COX, Robin y PERRY, Karen-Marie. Like a fish out of water: reconsidering disaster recovery and the role of place and social capital in community disaster resilience. American Journal of Community Psychology. 48(3-4): 395-411, diciembre 2011. DOI 10.1007/s10464-011-9427-0.

DAVIS, Mike. Ciudad de cuarzo: arqueología del futuro de los ángeles. Madrid, Lengua de Trapo. 2002. 387 p. ISBN 9788489618947.

DI MASSO, Andrés. Micropolitics of public space: on the contested limits of citizenship as a locational practice. Journal of Social Political Psychology. 3(2): 63-83, octubre 2015. DOI 10.5964/jspp.v3i2.322.

--- Public space in conflict: place meaning as contested interaction and ideological action. Barcelona, España, Universitat de Barcelona. 2009. Tesis de doctorado.

FRANCIS, Mark. Control as a dimension of publicspace quality. En: ALTMAN, Irwin; ZUBE, Ervin. Public places and spaces. New York, Plenum Press. 1989. p. 147-172. Human Behavior and Environment. ISBN 978-1-4684-5601-1.

FRANCIS, Mark. Urban open space: Designing for user needs. Washington, DC, Island Press. 2003. 96 p. ISBN 978-1559631136.

FRASER, Nancy. Rethinking the public sphere: A contribution to the critique of actually existing democracy. Social Text. (25/26): 56-80, 1990. DOI $10.2307 / 466240$.

GEHL, Jan. Ciudades para la gente. Buenos Aires, Infinito. 2014. 280 p. ISBN 9789879393802.
--- Public spaces and public life. City of Adelaide 2002. [En línea]. Parks and Leisure Australia. Julio 2002. ISBN 1876-702-672. [Fecha de consulta: 20 agosto 2015] Disponible en: http://www.parksleisure. com.au/documents/item/773.

GOBIERNO Regional de Antofagasta. Estrategia regional de desarrollo 2009-2020. [En línea]. 2008. [Fecha de consulta: 01 junio 2013] Disponible en: http://www.goreantofagasta.cl/attachments/article/17/Estrategia\%202010-2020.pdf

GUSTAFSON, Per. Meanings of place: everyday experience and theoretical conceptualizations. Journal of Environmental Psychology. 21(1): 5-16, marzo 2001. DOI 10.1006/jevp.2000.0185.

HARVEY, David. Ciudades rebeldes. Del derecho de la ciudad a la revolución urbana. Madrid, Akal. 2013. 240 p. ISBN 978-84-460-3799-6.

LARENAS, Jorge; SALGADO, Marcela y FUSTER, Xenia. Enfrentar los desastres socionaturales desde los capitales y recursos comunitarios: el caso de la erupción volcánica de Chaitén, Chile. Magallania. 43(3): 125-140, diciembre 2015. ISSN 0718-0209. DOI $10.4067 / S 0718-22442015000300010$.

LEFEBVRE, Henri. El derecho a la ciudad. Barcelona, Península. 1978. 169 p. ISBN 9788429709162.

LOW, Setha y ALTMAN, Irwin. Place attachment: a conceptual inquiry. En: ALTMAN, Irwin; LOW, Setha. Human Behavior and Environment Advances in Theory and Research. New York, Plenum Press. 1992. P. 1-12. ISBN 978-1-4684-8753-4. 
LYNCH, Kevin. A theory of good city form. Cambridge MA, MIT Press. 1984. 524 p. ISBN 978-0262620468.

MANZO, Lynne. For better or worse: exploring multiple dimensions of place meaning. Journal of Environmental Psychology. 25(1): 67-86, marzo 2005. DOI 10.1016/j.jenvp.2005.01.002.

MINISTERIO de Vivienda y Urbanismo. Reconstrucción urbana post 27F. Instrumentos de planificación y gestión territorial. [En línea]. MINVU. 2013. [Fecha de consulta: 20 mayo 2014]. Disponible en: http://www.minvu.cl/incjs/download.aspx?glb_ cod_nodo=20131008125015\&hdd_nom_ archivo=Reconstruccion\%20urbana\%2027F.pdf.

--- Plan de reconstrucción del borde costero, plan maestro Dichato. [En línea]. 2010. [Fecha de consulta: 01 junio 2013]. Disponible en: http:// www.minvu.cl/incjs/download.aspx?glb_ cod_nodo=20101207193158\&hdd_nom_ archivo=PRBC\%20Dichato.pdf.

MINISTERIO del Interior y Seguridad Pública. Diagnóstico estado de la reconstrucción erupción volcán Chaitén. [En línea]. Gobierno de Chile. 2014. [Fecha de consulta: 20 agosto 2015]. Disponible en: http://www.gob.cl/wp-content/uploads/2014/07/ Chaiten_V5.pdf.

MITCHELL, Don. The right to the city. Social justice and the fight for public space. New York, Guilford Press. 2003. 270 p. ISBN 978-1572308473.

--- The end of public space? People's Park, definitions of the public, and democracy. Annals of the Association of American Geographers. 85(1): 108-133, 1995. DOI 10.1111/j.1467-8306.1995. tb01797.xa.

POL, Enric. La apropiación del espacio. En: COGNICIÓN, representación y apropiación del espacio. Barcelona, Publicacions de la Universitat de Barcelona. 1996. p. 45-62. Collecció Monografies Psico-Socio-Ambientals, v. 9. ISBN 9788447515981.

RED Cross. World disasters report. Focus on culture and risk. [En línea]. Ginebra, Suiza, International Federation of Red Cross and Red Crescent Societies. 2014. 266 p. ISBN 978-92-9139-214-8. Disponible en http://www.ifrc.org/Global/Documents/Secretariat/201410/WDR\%202014.pdf.

RIVLIN, Leanne. Public spaces and public life in urban areas. En: NEARY, Susan, SYMES, Martin y BROWN, Frank. The urban experience: A peopleenvironment perspective. London, Chapman $\&$ Hall. 1994. p. 289-296. ISBN 0-419-20160-2.

SALGAD0, Marcela. Reconstrucción de la vida cotidiana. La cara invisibilizada tras el terremoto y tsunami del 2010. TS Cuadernos de Trabajo Social, Universidad de San Sebastián. (11): 34-42, 2014. ISSN 0719-6520. Disponible en: http://www.tscuadernosdetrabajosocial.cl/revista-11/pdf/ts114.pdf.

SENNETT, Richard. El declive del hombre público. Barcelona, Península. 1978. 437 p. ISBN 84-297-1445-6.

SMITH, Neil. El redimensionamiento de las ciudades: la globalización y el urbanismo neoliberal. 
En: HARVEY, David y SMITH, Neil. Capital financiero, propiedad inmobiliaria y cultura. Barcelona, MACBA/UAB. 2005. P. 59-75. ISBN 84-490-2409-9.

SORKIN, Michael. Variations on a theme park. The new American city and the end of public space. New York, Hill and Wang. 1992. 272 p. ISBN 978-0374523145.

STAEHELI, Lynn y MITCHELL, Donald. The people's property? Power, politics, and the public. New York, Routledge. 2008. 224 p. ISBN 978-0415955225.

STEDMAN, Richard. Is it really just a social construction? The contribution of the physical environment to sense of place. Society \& Natural Resources: An International Journal. 16(8): 671-685, 2003. DOI 10.1080/08941920309189.

STEDMAN, Richard; AMSDEN, Benoni; BECKLEY, Thomas y TIDBALL, Keith. Photo-based methods for understanding place meanings as foundations of attachment. En: MANZO, Lynne y DEVINEWRIGHT, Patrick. Place attachment. Advances in theory, methods and applications. London, Routledge. 2014. P. 113-124. ISBN 978-0-415-53811-4.

TAPIA, Ricardo. Acción del estado y acción comunitaria en la gestión de la vivienda post-erupción del volcán Chaitén, Chile: estrategias divergentes. Magallania. 43(3): 141-157, diciembre 2015. ISSN 07180209. DOI 10.4067/S0718-22442015000300011.

STRAUSS, Anselm y CORBIN, Juliet. Bases de la investigación cualitativa: técnicas y procedimientos para desarrollar la teoría fundamentada. Medellín, Colombia, Universidad de Antioquia. 2002. 341 p. ISBN 9789586556248.

TUAN, Yi-Fu. Space and place: humanistic perspective. En: BOARD, C., ed.; CHORLEY, R.J., ed.; HAGGETT, P., ed. y STODDART, D.R., ed. Progress in geography. New York, St. Martin's Press. 1974. p. 211-252

UGARTE, Ana María y SALGADO, Marcela. Sujetos en emergencia: acciones colectivas de resistencia y enfrentamiento del riesgo ante desastres; el caso de Chaitén, Chile. Revista INVI. 29(80): 143168, mayo 2014. ISSN 0718-8358. DOI 10.4067/ S0718-83582014000100006.

WILLIAMS, Daniel. Pluralities of place: A user's guide to place concepts, theories, and philosophies in natural resource management. En: KRUGER, Linda, HALL, Troy y STIEFEL, Maria. Understanding concepts of place in recreation research and management. Portland, U.S, Department of Agriculture, Forest Service, Pacific Northwest Research Station. 2008. P. 7-30. Disponible en: http://www. fs.fed.us/rm/pubs_other/pnw_gtr744_007_030. pdf.

WINKLER, María Inés; REYES, María Isabel; OLIVARES, Bárbara y BERROETA, Héctor. ¿Qué derecho tienen las comunidades ante las intervenciones comunitarias? [En línea]. Comunitaria. Marzo, 2016. Disponible en: http://comunitaria. 
cl/2016/03/derechos-de-las-comunidades-anteintervenciones-comunitarias/.

WISNER, Ben; BLAIKIE, Piers; CANNON, Terry y DAVIS, Ian. At risk. Natural hazards, people's vulnerability and disasters. New York, Routledge. 2003. 2a ed. 496 p. ISBN 978-0415252164.

WHYTE, William. The social life of small urban spaces. New York, Project for Public Space. 1980. 125 p. ISBN 978-0970632418.

ŽIŽEK, Slavoj. Ideología: Un mapa de la cuestión. Buenos Aires, Paidós. 1994. ISBN 978-9505575732. 